ISSN 2447-9071

doi $10.36414 /$ rbmc.v6i14.37
Contato para correspondência:

Lorraine Vieira Cruz

E-mail:

lorraine_vieiracruz@hotmail.com

Conflito de interesse: Não

Financiamento: Recursos próprios

Recebido: 04/04/2020

Aprovado: $14 / 04 / 2020$

\section{Associação entre obesidade e câncer gástrico}

\section{Association between obesity and gastric cancer}

Amanda Conceição Lopes ${ }^{1}$, Lorraine Vieira Cruz' ${ }^{1}$, Hermínio Maurício da Rocha Sobrinho 1,2

1 Pontifícia Universidade Católica de Goiás - PUC Goiás

${ }^{2}$ Universidade Estadual de Goiás - UEG

\section{Resumo}

O objetivo deste estudo foi descrever a associação dos principais fatores relacionados com a fisiopatologia da obesidade com o desenvolvimento do câncer gástrico. Consiste em uma revisão bibliográfica narrativa. Nesta pesquisa utilizou-se as bases de dados virtuais: Periódicos Capes, Biblioteca Virtual em Saúde e Pubmed. Foram incluídos 57 artigos publicados no período de 2005 a 2019 que apresentavam conteúdos compatíveis com os objetivos deste estudo. A obesidade está entre as doenças que mais crescem no mundo, o tratamento é inadequado e os distúrbios associados, incluindo câncer gástrico, apresentamaltamorbimortalidade. Aobesidade pode afetaramicrobiotaintestinal resultandoem uma disbiose, através da interação entre fatores nutricionais, endócrinos e metabólicos, que contribuem por ativar um processo de inflamação crônica de baixo grau levando ao aumento da proliferação celular e angiogênese e diminuição da apoptose celular, culminando no desenvolvimento de câncer gástrico. Dessa forma, é importante ressaltar que o tecido adiposo responde à estimulação de nutrientes extras via hiperplasia e hipertrofia de adipócitos. Com a hipertrofia dos adipócitos, o tecido adiposo torna-se hipoperfundido, o que cria áreas de microhipóxia, ativando as vias do fator de transcrição nuclear NFkB, aumentando a expressão de genes envolvidos na inflamação com maior liberação de citocinas. Assim, níveis elevados de adiponectina eleptina, esteroides sexuais, glicocorticoides, mediadores inflamatórios e fatores de crescimento semelhantes à insulina, podem resultar em estresse oxidativo tecidual e na transformação neoplásica das células gástricas. Énecessário um maior entendimento dos mecanismos da carcinogênese induzida pela obesidade para desenvolver métodos mais eficazes para prevenir ou tratarocâncergástrico. Umamaiorcompreensãodosmecanismosmolecularespodelevaràidentificação de novos alvos terapêuticos. Faz-se necessário estudos adicionais sobre os mecanismos envolvidos na carcinogênese gástrica, pois essa doença ainda apresenta altas taxas de morbimortalidade.

Palavras-Chave: Câncer Gástrico, Obesidade, Citocinas, Adipocinas, Imunopatologia.

\section{Abstract}

The objective of this research was to describe the association of the main factors related to the pathophysiology of obesity with the development of gastric cancer. This work constitutes a narrative bibliographic review. In this research, we used the virtual databases: Capes Periodicals, Virtual Health Library and Pubmed. 57 articles published in the period from 2005 to 2019 that included content compatible with the objectives of this study were included. Obesity is among the fastest growing diseases in the world, treatment is inadequate and the associated disorders, including gastric cancer, have high morbidity and mortality. Obesity can affect the intestinal microbiota resulting in dysbiosis, through the interaction between nutritional, endocrine and metabolic factors, which contribute to activate a process of low-grade chronic inflammation leading to increased cell proliferation and angiogenesis and decreased cell apoptosis, culminating in the development of gastric cancer. Thus, it is important to note that adipose tissue responds to the stimulation of extra nutrients via adipocyte hyperplasia and hypertrophy. With adipocyte hypertrophy, the adipose tissue becomes hypoperfused, which creates areas of microhypoxia, activating the nuclear transcription factor NFkB pathways, increasing the expression of genes involved in inflammation with greater release of cytokines. Thus, high levels of adiponectin and leptin, sex steroids, glucocorticoids, inflammatory mediators and insulin-like growth 
factors, can result in oxidative tissue stress and the neoplastic transformation of gastric cells. A greater understanding of themechanisms of obesity-induced carcinogenesis isneeded to developmoreeffective methods to prevent or treat gastric cancer. A greater understanding of the molecularmechanisms can lead to the identification of new therapeutic targets Additional studies on the mechanisms involved in gastric carcinogenesis are necessary, as this disease still has high rates of morbidity and mortality.

Keywords: Stomach Neoplasms, Obesity, Cytokines, Adipokines, Immunopathology.

\section{introdução}

O Câncer Gástrico (CG) é uma patologia de alta prevalência na população brasileira, apresenta altas taxas de mortalidade, configurando um problema de Saúde Pública. No Brasil, o CG é o quinto câncer mais frequente, excetuando-se o de pele (não melanoma), sendo no gênero masculino o terceiro tipo de câncer mais frequente e o quinto no gênero feminino'. Em ambos os gêneros a incidência aumenta a partir de 35 a 40 anos em proporções diferentes. De modo geral, as taxas de incidência são $2 \mathrm{a}$ 3 vezes maiores em homens do que em mulheres, gerando altos custos para o sistema público de saúde e para os pacientes ${ }^{1,2}$.

Os tumores gástricos se apresentam na forma de três tipos histológicos: adenocarcinoma (responsável por $95 \%$ dos tumores) linfoma, diagnosticado em cerca de $3 \%$ dos casos, e leiomiossarcoma (2\%), iniciado em tecidos que dão origem aos músculos e aos ossos ${ }^{3}$.

Apesar da etiologia não ser completamente conhecida, a carcinogênese gástrica surge como consequência de uma complexa interação entre o organismo e fatores ambientais e comportamentais ${ }^{4}$. A patogênese do CG se manifesta através de modificações na mucosa gástrica sob ação de fatores ambientais, endócrinos e imunológicos que induzem a substituição das células normais por células neoplásicas. Esse processo ocorre a longo prazo e sugere que os fatores de risco, como o hiperinsulinismo, resistência periférica a insulina, aumento de ácidos graxos livres, hiperlipidemia, estresse oxidativo e aumento de citocinas atuem por vários anos contribuindo para o desenvolvimento adenocarcinoma gástrico ${ }^{5}$.

Recentemente foi relatado que o desenvolvimento de adenocarcinoma de cárdia gástrica está fortemente associado à obesidade ${ }^{6,7}$, sugerindo assim, a possibilidade de outras causas além da infecção por H. pylori. Além disso, sabe-se que o tecido adiposo (TA) secreta hormônios, proteínas de fase aguda, adipocinas, fatores hemostáticos e hemodinâmicos e até mesmo fatores de crescimento que contribuem para o desenvolvimento do $\mathrm{CG}^{8}$.

O CG ainda permanece como uma doença desafiadora, uma vez que se mantém entre as principais causas de morte por neoplasia. A presença de lesão com apresentação insidiosa ou até mesmo assintomática contribui para o alto número de diagnósticos já em estágios avançados da doença, comprometendo o tratamento 9 . O objetivo deste estudo foi descrever a associação dos principais fatores relacionados com a fisiopatologia da obesidade com o desenvolvimento do adenocarcinoma gástrico.

\section{Métodos}

Esta pesquisa é caracterizada como revisão bibliográfica narrativa. Para a busca dos estudos foram utilizadas as bases de dados virtuais: Periódicos da Capes, Biblioteca Virtual em Saúde (BVS) e Pubmed/Medline, utilizando-se os seguintes termos e Descritores em Ciências da Saúde (DeCS), com múltiplas combinações: Stomach Neoplasms, Obesity, Cytokines, Adipokines, Immunopathology and Immune Response nos idiomas português, inglês e espanhol, publicados no período do ano 2005 ao ano de 2019. Foram incluídos um total de 57 artigos disponíveis, com texto completo, nas bases de dados eletrônicas utilizadas e que apresentaram conteúdos compatíveis com os objetivos propostos. Foram excluídos do estudo os trabalhos publicados fora do período temporal mencionado, os duplicados, os que não estavam disponíveis na íntegra nas bases de dados e aqueles não condizentes com os objetivos propostos.

Figura 1: Algoritmo adotado para a busca e seleção de artigos relacionados com a associação entre a obesidade e o desenvolvimento de câncer gástrico.

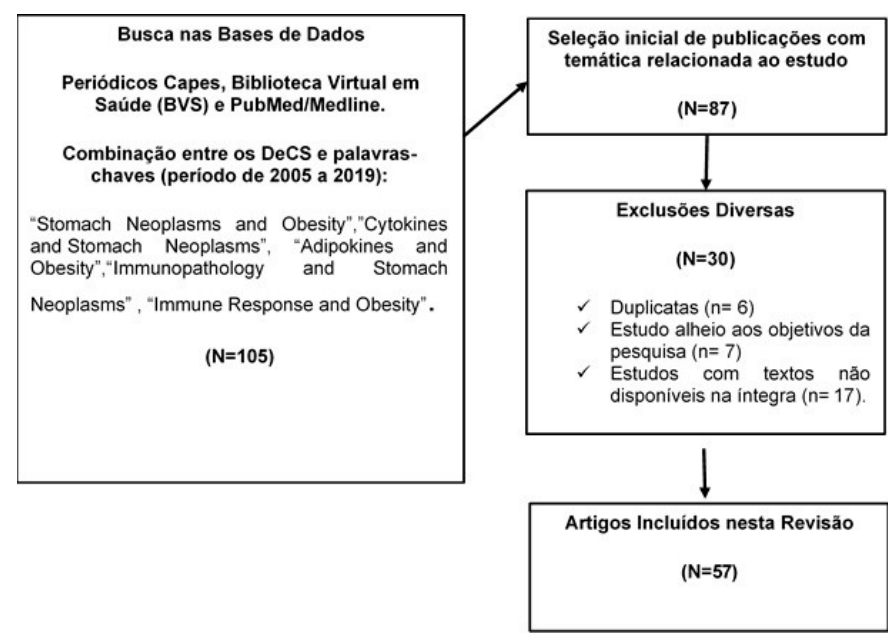


Resultados e Discussão

O adenocarcinoma gástrico é uma doença multifatorial, tanto fatores ambientais quanto genéticos têm importante papel em sua etiologia ${ }^{10}$.

A obesidade tem sido associada ao desenvolvimento de diferentes tipos de câncer, estando relacionada a 10\% de todas as mortes oncológicas em não fumantes e presente em aproximadamente $14 \%$ de todas as mortes por câncer em homens e $20 \%$ em mulheres. Estudo aponta que a cada $5 \mathrm{~kg} / \mathrm{m} 2$ de aumento no IMC aumenta o risco de mortalidade por neoplasias em até $10 \%{ }^{11}$.

\section{A obesidade e os principais mecanismos fisiopatológicos para o desenvolvimento do Câncer Gástrico}

A obesidade representa um estado patológico durante o qual o tecido adiposo sofre uma expansão maciça, principalmente, pela hipertrofia adipocitária que prejudica a circulação sanguínea tecidual, levando à hipóxia, inflamação e fibrose ${ }^{12}$. Além disso, o acúmulo patológico de gordura tecidual está associado ao aumento do metabolismo oxidativo celular e ao mal funcionamento da resposta de desdobramento de proteínas que prejudicam as principais funções dos adipócitos na regulação do armazenamento lipídico e da secreção de adipocinas ${ }^{13}$.

Os possíveis mecanismos fisiopatológicos responsáveis pela associação entre obesidade e câncer incluem a distribuição da gordura corporal, processo inflamatório, alterações imunológicas, estresse oxidativo celular, alterações nutricionais, disbiose e alterações nos padrões hormonais, envolvendo o eixo InsulinaIGF e hormônios esteroides, adipocinas produzidas no tecido adiposo visceral. Esse conjunto de fatores promove a resistência insulínica e aumento na produção de insulina pelo pâncreas para compensar o metabolismo da glicose. Alterações endócrinas, imunológicas e metabólicas favorecem o crescimento tumoral a partir de seus efeitos mitogênicos, anti-apoptóticos e angiogênicos, atuando diretamente sobre os tecidos ou, indiretamente, por mudanças no metabolismo corporal ${ }^{14,15}$. Entretanto, as explicações sobre estas associações e mecanismos fisiopatológicos ainda são escassas quanto a essas hipóteses ${ }^{11}$.

É importante ressaltar que a hiperinsulinemia reduz a produção de IGFBP-1 (Insulin-like Growth Factor Binding Proteins) e IGFBP-2, que normalmente se ligam ao Fator de Crescimento de Insulina Símile-1 (IGF-1-Insulin-like Growth Factor-1) inibindo sua ação, dessa forma, haverá em aumento nos níveis de IGF-1 livre, bioativo. Esta somatomedina, junto à insulina, se liga aos seus receptores, resultando na fosforilação de proteínas IRS, que ativam a cascata de sinalização intracelular, representada pelas vias fosfoinositol-3-quinase (PI3K-Akt) e proteína quinase ativada pelo mitógeno (MAP quinase ou MAPK), ambas envolvidas no processo da carcinogênese ${ }^{16}$.

\section{Fatores Endócrinos}

A obesidade está diretamente relacionada ao desenvolvimento do CG, o mecanismo patológico não é claramente descrito. Contudo, sabe-se que aumenta o risco de refluxo gastresofágico e de resistência periférica à insulina. Além disso, apresenta níveis elevados de adiponectina e leptina, esteroides sexuais, glicocorticoides, mediadores inflamatórios e a presença de fatores de crescimento semelhantes à insulina. Resultado desse estresse oxidativo e a transformação neoplásica das células gástricas ${ }^{17,18}$.

O tecido adiposo é o principal local da síntese periférica de estrogênio. A obesidade causa um aumento na produção e circulação de estrógenos livres ativos (principalmente estradiol), andrógenos e testosterona, e uma redução nos níveis da globulina ligada ao hormônio sexual. $O$ estradiol aumenta a sensibilidade aos receptores de insulina e fator de crescimento semelhante à insulina (IGF) ${ }^{15}$.

A obesidade aumenta as concentrações de insulina, IGF1 e IGF2, que por sua vez reduzem a apoptose e estimulam o crescimento celular. Com o aumento da adiposidade ocorre também o aumento da produção de citocinas pró-inflamatórias tais como a interleucina-6 (IL-6) e Interleucina-8 (IL-8), fator de necrose tumoral (TNF) e proteína $C$ reativa ${ }^{15}$.

Portanto, os adipócitos da gordura visceral formam um órgão endócrino ativo que secreta adipocinas como a adiponectina. A adiponectina aumenta a sensibilidade à insulina $e$ pode ter um efeito anti-inflamatório e anticâncer ${ }^{15}$. Também tem efeitos antiproliferativos e angiogênicos. Muitos cânceres expressam receptores de adiponectina, incluindo tumores gástricos ${ }^{19}$.

O estado de hiperinsulinismo seria o responsável pela estimulação da $\beta$-catenina, uma via de sinalização precoce em neoplasias, que promove a inibição da glicogênio sintase $3 \beta$ e a ativação de oncogenes Ras-MAPK, além de induzir a proteólise de IGFBP-3, o que poderia reduzir a afinidade de IGF-1 aos fragmentos de IGFBP-3, aumentando a liberação de IGF-1 livre ${ }^{16}$.

A estimulação exógena de células cancerígenas gástricas, relacionadas com IGF1 e IGF2 levam à proliferação celular, indicando que o sistema IGF está ativo e pode desempenhar um papel significativo na fisiopatologia e tumorigênese da neoplasia gástrica. O eixo IGF-IGFR-IGFBP consiste em ligantes, a insulina IGF1 ou a IGF2 se liga a IGF1R (uma tirosina quinase receptora) e ativa a sinalização através do substrato receptor da insulina (IRS), para regular a proliferação celular ou a sobrevivência e progressão do ciclo celular. A maioria das ações conhecidas dos IGFs é exercida mediante sua ligação ao receptor tipo 1 (IGF-1R), não sendo ainda claro o papel fisiológico do receptor tipo 2 (IGF2R). Há indícios de que o IGF-2R possa participar da remoção 
do IGF-2 do ambiente extracelular. Assim, múltiplas vias de sinalização, entre elas, a via da fosfoinositol-3-quinase (PI3K) e da proteína quinase ativada pelo mitógeno (MAP quinase), são ativadas pela interação entre os IGFs e seus receptores. Essas vias estão envolvidas no transporte de glicose, na regulação da síntese de glicogênio e de uma variedade de reguladores de sobrevida celular, além de inibição da apoptose ${ }^{20}$. O IGF1 estimula a divisão celular e inibe a apoptose, podendo, portanto, contribuir para o desenvolvimento e a metástase do câncer ${ }^{19}$.

A hiperglicemia também pode aumentar a disponibilidade de nutrientes para as células cancerígenas, que metabolizam a glicose pelo efeito Warburg, em que as células cancerígenas produzem energia por uma alta taxa de glicólise, seguida pela fermentação com ácido lático no citosol, em vez de uma taxa comparativamente baixa de glicólise, seguida de oxidação do piruvato nas mitocôndrias ${ }^{19}$.

A obesidade aumenta o tamanho dos adipócitos, levando à necrose e subsequente acúmulo de macrófagos ativados. Estes secretam mediadores inflamatórios, incluindo as citocinas pró-inflacumatórias IL-6, MCP1 e TNF-a, que estão implicadas no desenvolvimento da resistência à insulina. Essa inflamação é acompanhada pela regulação positiva de citocinas antiinflamatórias IL-10, IL-4 e TGF- $\beta$. $O$ tecido adiposo em pacientes obesos tem um repertório restrito de receptores de células $\mathrm{T}$, contendo menos células T reguladoras (Linfócitos T reg) do que o tecido adiposo em indivíduos magros. A atividade alterada das células $T$ reguladoras pode aumentar a inflamação no tecido adiposo para promover a carcinogênese ${ }^{19}$. Os principais mecanismos fisiopatológicos da associação de obesidade com o câncer gástrico estão esquematizados, à seguir, na figura 2 .

Figura 2: Principais mecanismos fisiopatológicos da associação de obesidade com o câncer gástrico.

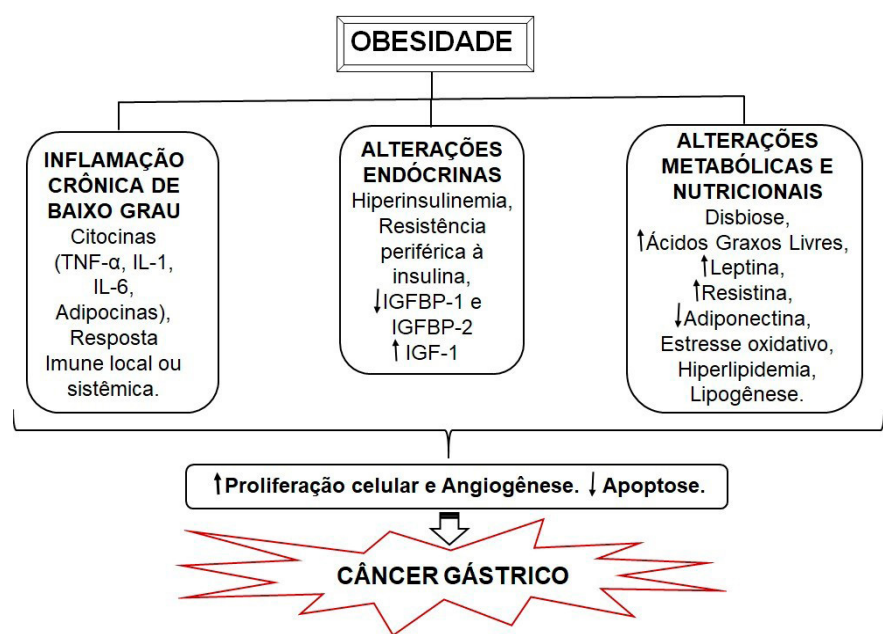

A obesidade está associada à inflamação crônica de baixo grau (também chamada meta-inflamação), caracterizada por produção anormal de citocinas, ativação imune e aumento da sinalização inflamatória ${ }^{19}$.

As citocinas pró-inflamatórias superexpressas na obesidade são consideradas o elo entre obesidade e inflamação. $O$ tecido adiposo responde à estimulação de nutrientes extras via hiperplasia e hipertrofia de adipócitos ${ }^{21}$. Com a hipertrofia dos adipócitos, o tecido adiposo torna-se hipoperfundido, o que cria áreas de microhipóxia, ativando células imunológicas, especialmente, pela via do fator de transcrição nuclear NFkB, aumentando a expressão de genes envolvidos na inflamação com maior liberação de citocinas e recrutamento de macrófagos para o tecido lesado 22 .

As adipocinas são peptídeos bioativos sintetizados e secretados por adipócitos, entre elas tem se a leptina (LEP) um hormônio que está associado com o sinal de saciedade ${ }^{23}$. A sequência primária de aminoácidos dos receptores da LEP indica semelhança a família de citocinas helicoidais, tais como a Interleucina-2 (IL-2), Interleucina-12 (IL-12) e fator estimulador de colônias de granulócitos (G-CSF), isso justifica a forte relação entre a leptina e a resposta inflamatória ${ }^{24}$.

A leptina está associada positivamente à resistência à insulina e à obesidade ${ }^{15}$. Além disso, estimula a proliferação celular, a migração de células tumorais, inibe a apoptose e estimula a liberação de citocinas dos macrófagos. A leptina produzida pelo tecido adiposo pode afetar os tumores adjacentes, está envolvida na angiogênese e pode ativar a aromatase no tecido adiposo. Além do mais, como a leptina inibe a atividade das células T reguladoras (Treg), ela pode regular a vigilância imunológica dos cânceres gastrointestinais ${ }^{19}$.

O hormônio grelina desempenha um papel na regulação do apetite, no metabolismo de ácidos graxos e promove o armazenamento de gordura. Verificou-se que está intimamente associado ao risco de câncer gástrico e adenocarcinoma do esôfago ${ }^{15}$.

A resistina (RES) é um peptídeo encontrado em altos níveis em obesos, sendo considerada uma molécula pró-inflamatória e que está relacionada a complicações do diabetes, estando envolvida também na proliferação dos adipócitos e angiogênese. Assim, a grelina está envolvida no processo inflamatório por ser um forte regulador da secreção celular de IL-6 e do TNF-a pela ativação do fator nuclear kappa B (NF- kB) ${ }^{25}$.

$O$ TNF-a é uma citocina que está associada à resistência à insulina, sendo sua produção influenciada positivamente por triglicerídeos (TG) e ácidos graxos. Assim, a ausência de expressão de TNF-a tem efeito protetor quanto ao desenvolvimento de resistência insulínica em estudo realizado em murinos ${ }^{26}$. 
A IL-1 $\beta$ é capaz de induzir inflamação sistêmica a partir da ativação da ciclooxigenase-2. Essa citocina encontra-se elevada em indivíduos obesos e observou-se que a sua secreção é maior por macrófagos infiltrados no tecido adiposo e menor por adipócitos ${ }^{27}$.

A adiponectina (APN) é uma adipocina capaz de regular a expressão de citocinas pró-inflamatórias induzindo a produção de IL-10 (citocina anti-inflamatória) e inibindo o TNF-a, tendo, portanto, um efeito anti-inflamatório que contribui para a proteção contra os efeitos do estresse na doença metabólica e na obesidade ${ }^{28}$. De forma oposta à maioria das adipocinas, a APN se encontra em níveis diminuídos em indivíduos obesos ${ }^{29}$.

Estudos clínicos estabeleceram a correlação entre baixos níveis de APN e cânceres relacionados à obesidade ${ }^{30}$. Os dados correlacionam APN sérico baixo com número e tamanho aumentados de focos tumorais ${ }^{31}$ e estágio elevado de invasão e malignidade ${ }^{32}$. De fato, os baixos níveis plasmáticos de APN se associam a cânceres relacionados à obesidade e hormônios esteroides sexuais, enquanto concentrações séricas aumentadas de APN parecem indicar status inflamatório e estágios avançados de malignidade ${ }^{30,33}$.

Várias linhas de evidência sugerem que o aumento dos níveis circulantes de APN ou a ativação de vias de sinalização induzidas por APN podem neutralizar disfunções metabólicas e retardar a progressão do câncer em modelos experimentais ${ }^{34,35,36}$.

É importante ressaltar o papel de ativação da AMPK (proteína quinase ativada por AMP) na homeostase energética. Assim, uma vez ativada, AMPK exerce efeitos sobre o metabolismo da glicose e lipídios, atuando em diversos órgãos. A ativação da AMPK parece suprimir a resposta inflamatória no tecido adiposo de indivíduos obesos por meio da inibição da síntese de citocinas pró-inflamatórias como TNF-a, IL-6 e IL-837. AMPK regula também os níveis de leptina e adiponectina ${ }^{38}$.

\section{Fatores Nutricionais}

Há uma correlação do potencial carcinogênico de alimentos ricos em carboidratos com a exposição da mucosa gástrica a estes alimentos, os quais podem induzir um estágio de gastrite crônica. Quando a gastrite crônica não é tratada ocorre a formação de nitrosaminas, as quais apresentam atividade carcinogênica, podendo evoluir para o desenvolvimento de um carcinoma gástrico ${ }^{39}$. Os principais fatores de risco nutricionais que podem contribuir para o desenvolvimento de câncer gástrico são apresentados no quadro 1.
Quadro 1: Principais fatores de risco nutricionais relacionados com o desenvolvimento do câncer gástrico.

Fatores nutricionais

Alta ingesta de nitratos/nitritos.

Alimentos que favorecem a formação de nitrosaminas.

Alta ingesta de sal.

Alta ingesta de amido.

Alimentos mal conservados.

Fonte: RESENDE et $\mathrm{al}^{40}$.

Diversos fatores podem desencadear a resposta inflamatória no tecido adiposo em condições de sobrecarga nutricional, dentre eles é importante ressaltar a hipóxia ${ }^{41}$, o estresse oxidativo celular ${ }^{42}$ e ativação celular por ácidos graxos saturados, por um mecanismo dependente da ativação de receptor Toll-like 4 (TLR4) ${ }^{43}$.

OTLR4 é membro de uma família de receptores de reconhecimento de padrões moleculares antigênicos presentes em diferentes células e tecidos humanos e envolvido na resposta imune inata. Estudo demonstra que ácidos graxos saturados também podem ativar o TLR4. Desse modo, esse mecanismo de ativação tem sido considerado um importante fator de ligação entre inflamação e resistência à insulina em condições de obesidade e sobrecarga nutricional ${ }^{44}$.

Desse modo, a ativação da resposta inflamatória por uma sobrecarga nutricional pode tornar-se um fator promotor para o desenvolvimento da resistência à insulina e, consequentemente, disfunção do tecido adiposo ${ }^{45}$.

Os ácidos graxos modulam o crescimento das células da mucosa gastrointestinal e podem induzir danos ao $D^{2} A^{46} \mathrm{e}$ contribuem para o início e a progressão do desenvolvimento de vários tipos de cânceres ${ }^{47-49}$. As principais contribuições dos ácidos graxos para o desenvolvimento do câncer gástrico são apresentadas no quadro 2 , a seguir.

Quadro 2: A contribuição dos ácidos graxos para o desenvolvimento do câncer gástrico.

\begin{tabular}{ll}
\hline Ácidos Graxos & Mecanismo \\
\hline $\begin{array}{l}\text { Ácido Palmítico e os ácidos graxos } \\
\text { saturados }\end{array}$ & $\begin{array}{l}\text { Promovem a metástase das células do CG } \\
\text { através da cascata da proteína } 5 \text { de ligação a } \\
\text { ácidos graxos. }\end{array}$ \\
Ácido Oleico & $\begin{array}{l}\text { Aumenta a invasão das células CG pela via } \\
\text { PI3K-Akt. }\end{array}$ \\
\hline
\end{tabular}

Fonte: XIANG et $\mathrm{al}^{50}$. 
Por outro lado, os ácidos graxos poli-insaturados N-3 têm a capacidade de suprimir a produção de citocinas pró-inflamatórias em pacientes com CG $\mathrm{CG}^{51}$. Outro aspecto protetor é a dieta mediterrânea, caracterizada pelo aumento do consumo de azeite de oliva, que pode prevenir o câncer gástrico ${ }^{52}$.

É fato que os conservantes adicionados aos embutidos contribuem para o aparecimento de malignidades gastrointestinais, pois quando estes chegam ao estômago transformamse em nitrosaminas, substâncias cancerígenas responsáveis por alterações celulares que podem levar ao desenvolvimento de câncer. Enquanto os defumados contêm hidrocarbonetos policíclicos aromáticos e alcatrão que apresentam ação carcinogênica conhecida ${ }^{53}$.

\section{Disbiose}

Sabe-se que a nutrição pode afetar a microbiota intestinal e o microbioma, o que contribui amplamente para doenças sistêmicas ${ }^{54,55}$. Dessa forma, a disbiose, um desequilíbrio do microbioma induzido pela alimentação, especialmente por alimentos ricos em gordura, está associada à malignidade gastrointestinal ${ }^{56}$.

Muito pouco se sabe sobre a contribuição da microbiota intestinal para o desenvolvimento de neoplasias digestivas. Os microrganismos entéricos podem promover a carcinogênese por diferentes mecanismos: 1) indução de inflamação; 2) aumento da proliferação celular; 3) alteração na dinâmica das células tronco; 4) produção de algumas substâncias como o butirato, capaz de afetar a integridade do DNA e a regulação imunológica ${ }^{57}$.

\section{Conclusão}

A obesidade está entre as doenças que mais crescem no mundo, o tratamento é inadequado e os distúrbios associados, incluindo câncer gástrico, apresentam alta morbimortalidade. Os possíveis mecanismos responsáveis pela associação entre obesidade e câncer incluem a distribuição da gordura corporal e alterações nos padrões hormonais, envolvendo o eixo Insulina-IGF, estrógenos e progesterona, adipocinas e citocinas produzidas no tecido adiposo visceral.

Diante das evidências apresentadas neste estudo, observa-se que o tecido adiposo em expansão pode ter uma contribuição clinicamente relevante no desenvolvimento do câncer gástrico. É necessário um maior entendimento dos mecanismos da carcinogênese induzida pela obesidade para desenvolver métodos para prevenir ou tratar o câncer gástrico. Uma maior compreensão dos mecanismos moleculares presentes na obesidade pode levar à identificação de novos alvos terapêuticos. $\mathrm{O}$ estudo dos fatores que relacionam a obesidade com a carcinogênese desta neoplasia podem trazer subsídios futuros para terapias mais eficazes para o CG, assim como, proporcionar novas medidas que visam à prevenção para modificar a história natural do adenocarcinoma gástrico, pois essa neoplasia ainda apresenta altas taxas de morbimortalidade, devido a sua clínica ausente ou inespecífica somada ao diagnóstico tardio. Desse modo, faz-se necessário maior atenção na prevenção dos fatores de risco e principalmente da obesidade.

Dada a falta de terapias efetivas atuais para controle ou redução de peso além da cirurgia bariátrica, a pesquisa e os esforços clínicos se concentrarão nas conexões entre o aumento da massa celular adiposa e a carcinogênese gastrointestinal. Estudos adicionais dos fatores hormonais, inflamatórios, genéticos e alimentares que contribuem para o desenvolvimento e progressão do câncer gástrico nos ajudarão a entender o papel da obesidade nesses processos.

\section{Referências}

1. Brasil. Ministério da Saúde. Instituto Nacional de Câncer. Estimativa 2016: Incidência de câncer no Brasil. Rio de Janeiro: INCA; 2015.

2. Ferlay J, Shin RH, Bray F, Forman D, Mathers C, Parkin DM. Estimates of worldwide burden of cancer in 2008: GLOBOCAN 2008. Int J Cancer. 2010;127(12):2893-917.

3. Brasil. Ministério da Saúde. Instituto Nacional de Câncer. Estimativa 2014/2015: Incidência de câncer no Brasil. Rio de Janeiro: INCA. 2014;68:38-39.

4. Ang TL, Fock KM. Clinical epidemiology of gastric cancer. Singapore Med J. 2014;12(55):621-628.

5. Silva VCS, Felício DC. Fatores de risco para o câncer gástrico em grupos de classe socioeconômico baixa: revisão literária. Revista de Iniciação Científicada Universidade Vale do Rio Verde. 2016; 6(1):3-10.

6. Cho Y, et al. Higher prevalence of obesity in gastric cardia adenocarcinoma compared to gastric non-cardia adenocarcinoma. Dig Dis Sci. 2012;57(10):2687-92.

7. O'Doherty MG, Freedman ND, Hollenbeck AR, Schatzkin A, Abnet CC. A prospective cohort study of obesity and risk of oesophageal and gastric adenocarcinoma in the nih-aarp diet and health study. Gut. 2012;61(9):1261-8.

8. Proença ARG, et al. New concepts in white adipose tissue physiology. Brazilian journal of medical and biological research. 2014;47(3):192- 205.

9. Todescatto DA, et al. Câncer Gástrico. Portal de Revistas Acta Minha BVS. Organização Pan Americana da Saúde. 2017; 38(6) 38. 
10. Karimi P ,Islami F, Anandasabapathy S, Freedman ND, Kamangar F. Gastric Cancer: Descriptive Epidemiology, Risk Factors, Screening, and Prevention. Cancer Epidemiology Biomarkers \& Prevention. 2014;23(5):700-13.

11. Ferraz $A A B$, Siqueira LT. Obesidade e Câncer. GASTRÃO 2015. Anais do Gastrão .2015; p.001. Disponível em: https://www.ufmt.br/ppgcs/images/uploads/editais/ papers2019/Linha1/Obesidadeecancer. Acessado em 10/01/2020.

12. Sun K, Kusminski CM, Scherer PE. Adipose tissue remodeling and obesity. J Clin Invest. 2011;121(6):20942101.

13. Trayhurn P. Hypoxia and adipose tissue function and dysfunction in obesity. 2013;93(1):1-21.

14. Vongsuvanh R, George J, Qiao L, van der Poorten D. Visceral adiposity in gastrointestinal and hepatic carcinogenesis. Cancer Lett. 2013;330(1):1-10.

15. Svatetz CAG, Arnô AG. Obesity and cancer: "Dangerous friendship. Ver. Med Clin (Barc). 2015;145(1):24-30.

16. Renehan AG, Frystyk J, Flyvbjerg A. Obesity and cancer risk: the role of the insulin-IGF axis. Trends Endocrinol Metab. 2006;17(8):328-36.

17. Kuriyama $S$, et al. Obesity and risk of cancer in Japan. Int J Cancer, 2005;113(1):148-57.

18. Sjodahl K, et al. Body mass and physical activity and risk of gastric cancer in a population-based cohort study in Norway. Cancer Epidemiol Biomarkers Prev. 2008;17(1):135-40.

19. Alemán JO, Eusebi LH, Ricciardiello L, Patidar K, Sanyal AJ, Holt PR. Mechanisms of Obesityinduced Gastrointestinal Neoplasia.Gastroenterology.2014;146(2):357-373.

20. Kashyap MK. Role of insulin-like growth factor-binding proteins in the pathophysiology and tumorigenesis of gastroesophageal cancers. Tumor Biology. 2015;36(11):8247-57.

21. Hotamisligil GS. Inflamação e distúrbios metabólicos. Natureza. 2006;444(7121): 860-867.

22. Schenk S, Saberi M, Olefsky JM. Insulin sensitivity: modulation by nutrients and inflammation. J Clin Invest . 2008;118(9):2992-3002.

23. Krysiak R, Handzlik-Orlik L, Okopien B. The role of adipokines in connective tissue diseases. European journal of nutrition. 2012;51(5):513-28.

24. Paz-Filho G, Wong ML, Licinio J. Ten years of leptin replacement therapy. Obesity Reviews. 2011;12(5):315-23.

25. Zhang L, Curhan GC, Forman JP. Plasma resistin levels associate with risk for hypertension among non- diabetic women. Journal of the American Society of Nephrology: JASN. 2010;21(7):1185-91.

26. Esteves D, Guimar D, Sardinha C. Adipokines: a new view of adipose tissue. Revista de Nutrição. 2007;20(5):549-559.

27. Fain JN. Release of Interleukins and Other Inflammatory Cytokines by Human Adipose Tissue Is Enhanced in Obesity and Primarily due to the Nonfat Cells. Vitamins and Hormones. 2006;74:443-77.

28. Silveira MR, Frollini AB, Verlengia R, Cavaglieri CR. Correlation between obesity, adipokines and the immune system. Revista Brasileira Desempenho Humano. 2009;11(4):466- 472.

29. Kim JY, et al. Prospective study of serum adiponectin and incident metabolic syndrome:The ARIRANG study. Diabetes Care. 2013;36(6):1547-53.

30. Dalamaga M, Diakopoulos KN, Mantzoros CS. The role of adiponectin in cancer: a review of current evidence. Endocr Rev. 2012;33(4):547-94.

31. Otake, AH. Papel de dissialogangliosídios na proliferação e morte celular induzida de melanócitos e melanomas in vitro; 2005. Tese (doutorado). Faculdade de Medicina da Universidade de São Paulo. Departameto de Radiologia.

32. Ishikawa F .Development of functional human blood and immune systems in NOD/SCID/IL2 receptor $\gamma$ chainnull mice. 2005;106(5):1565-73.

33. Izadi V, Farabad E, Azadbakht L. Serum adiponectin level and different kinds of cancer: a review of recent evidence. ISRN Oncol. 2012;2012:982769.

34. Jarde T, Perrier S, Vasson MP, Caldefie-Chézet F. Molecular mechanisms of leptin and adiponectin in breast cancer. Eur J Cancer. 2011;47(1):33-43.

35. Vansaun M.N. Molecular pathways: adiponectin and leptin signaling in cancer. Clin Cancer Res. 2013;19(8):1926-32.

36. Grossmann ME, Cleary MP. The balance between leptin and adiponectin in the control of carcinogenesis - focus on mammary tumorigenesis. Biochimie. 2012;94(10):2164-71.

37. Lihn AS, Pedersen SB, Lund S, Richelsen B.. The antidiabetic AMPK activator AICAR reduces IL-6 and IL-8 in human adipose tissue and skeletal muscle cells. Mol Cell Endocrinol. 2008;292(1-2):36-41.

38. Bijland S, Mancini SJ, Salt IP. Role of AMP-activated protein kinase in adipose tissue metabolism and inflammation. Clin Sci (Lond). 2013;124(8):491-507.

39. Mendonça R X. Agliardo LC, Ribeiro RL Câncer Gástrico: 
A importância da terapia nutricional. Saúde e Amb. Ver., 2008;3(2):7-19.

40. Resende ALS, Mattos IE, Koifman S. Dieta e câncer gástrico: aspectos históricos associados ao padrão de consumo alimentar no estado do Pará. Rev. Nutr. 2006;19(4):511-519.

41. Schuster D P. Obesity and the development of type 2 diabetes: the effects of fatty tissue inflammation. Diabetes Metab Syndr Obes. 2010; 3: 253-262.

42. Qatanani M, Lazar M A. Mechanisms of obesityassociated insulin resistance: many choices on the menu. Genes Dev. 2007;21(12):1443- 1455.

43. Poulain-Godefroy O. et al. Inflammatory role of Tolllike receptors in human and murine adipose tissue. Mediators Inflamm. 2010;2010:823486.

44. Erridge C, Samani NJ. Saturated fatty acids do not directly stimulate Toll-like receptor signaling. Arterioscler Thromb Vasc Biol. 2009;29(11):1944-9.

45. Ashida H, Yamashita Y, Fukuda I. Department of Applied Chemistry in Bioscience Biochemistry Frontiers. Kobe University. Disponível em: http://www.ans. kobe-u.ac.jp/english/gakka/seibutsu/seibutu1.html. Acessado 03/12/20.

46. Zeng L. et al. Saturated fatty acids modulate cell response to DNA damage: Implication for their role in tumorigenesis. PLoS ONE. 2008;3(6):2329.

47. Camarda R, et al. Inhibition of fatty acid oxidation as a therapy for MYC-overexpressing triple-negative breast cancer. Nat. Med.2016;22(4):427-32.

48. Hashimoto $S$, et al. Lysophosphatidic acid activates Arf6 to promote the mesenchymal malignancy of renal cancer. Nat. Commun. 2016; 7:10656.

49. Jeon SM, Chandel NS, Hay N. AMPK regulates NADPH homeostasis to promote tumour cell survival during energy stress. Nature. 2012;485(7400):661-5.

50. Xiang $F$, et al. Omental adipocytes enhance the invasiveness of gastric cancer cells by oleic acid-induced activation of the PI3K-Akt signaling pathway. Int. J. Biochem. Cell Biol. 2017;84:14-21.

51. Mocellin MC, Fernandes R, Chagas TR, Trindade EBSM. A meta-analysis of $n-3$ polyunsaturated fatty acids eff ects on circulating acute-phase protein and cytokines in gastric cancer. Clin. Nutr. 2018;37(3):840-850.

52. Castello A, et al. High adherence to the Western, Prudent, and Mediterranean dietary patterns and risk of gastric adenocarcinoma:MCC-Spain study.Gastric Cancer. 2018;21(3):372-382.

53. Baú FC, Huth A. Fatores de risco que contribuem para desenvolvimento do câncer gástrico e de esôfago. Revista Contexto e Saúde ljuí. 2011;11(21):16-24.

54. Sittipo $P$, et al. Intestinal microbiota and the immune system in metabolic diseases. J. Microbiol. 2018;56:154-162.

55. Ley RE, et al. Obesity alters gut microbial ecology. Proc. Natl. Acad Sci. USA. 2005;102(31):11070-5.

56. Carding S, Verbeke K, Vipond DT, Corfe BM, Owen LJ. Dysbiosis of the gut microbiota in disease. Microb. Ecol. Health Dis. 2015;26:10.3402 / mehd.v26.26191.

57. Passos MCF, Moraes-Filho JP. Intestinal microbiota in digestive diseases. Arq Gastroenterol 2017;54(3) 255-262. 